\title{
A cerebral abscess at first internist glance
}

\author{
Rita Ribeiro, ${ }^{1}$ Catarina Patrício, ${ }^{1}$ Margarida Moura Valejo Coelho, ${ }^{2}$ Vítor Brotas ${ }^{1}$
}

${ }^{1}$ Department of Internal Medicine, Hospital de Santo António dos Capuchos, Lisboa, Portugal

${ }^{2}$ Department of Dermatology, Hospital de Santo António dos Capuchos, Lisboa, Portugal

\section{Correspondence to}

Dr Catarina Patrício,

ana.catarina.lp@gmail.com

Accepted 12 November 2015

\section{SUMMARY}

A 73-year-old man was transferred to the neurosurgery ward, with a presumptive diagnosis of cerebral abscess. The case was also discussed with the internists as internal medicine consultants. The neurosurgeons pointed out a right temporal lobe abscedated lesion on $\mathrm{CT}$, but we noticed that the hypodense attenuation that usually surrounds the abscess wall (vasogenic oedema) extended to a broader, well-delimitated area, suggesting medium cerebral artery territory. The patient had leftsided hemiplegia with a confusional state and low-grade fever. Considering possible haematogenous dissemination, an echocardiography was performed, confirming mitral endocarditis. Blood cultures and aspirated pus isolated Escherichia coli. Investigating the patient's medical history, we learned he had been submitted to bladder catheterisation 7 weeks before for acute urinary retention due to benign prostatic hyperplasia, and empirically medicated for urinary tract infection. E. coli had also been isolated in a urine specimen at the time. The clinical history of the patient cancelled the pathogenesis of cerebral abscess.

\section{BACKGROUND}

Haematogenous cerebral Escherichia coli abscess is rare in adults. Brain abscess is a focal pus collection within the brain parenchyma, and the consequence of several infectious processes and agents. Clinical presentation tends to be non-specific, resulting in frequent diagnostic delay and, consequently, poorer prognosis. Although contiguous infection accounts for the majority of cases, haematogenous dissemination should always be considered, especially in situations where the clinical picture is not clear. Radiological and microbiological examinations give important clues about the aetiology of brain abscesses. Cerebral abscess and endocarditis can be serious complications of common and apparently innocent infections. Complicated urinary tract infections (UTIs) need to be treated aggressively as they can disseminate to cause potentially fatal results. In this setting, important bacteraemia can arise even with minor procedures.

\section{CASE PRESENTATION}

A 73-year-old man was transferred from another hospital to our neurosurgery ward, with the suspicion of a cerebral abscess. Our team of consultant internists was included in the initial evaluation of the patient. While the neurosurgeons focused on the abscedated mass and its attendant medical and surgical approaches, we emphasised that the CT scan showed a hypodensity attenuation surrounding not only the abscess wall, but also extending to a well-delimitated area suggestive of medium cerebral artery (MCA) territory. We wondered if this could be the result of a previous underlying ischaemic event, and not simply perilesional oedema. The neuroradiology expert confirmed the following findings: presence of a nucleobasal rounded lesion with mass effect; a multiloculated nucleobasal mass with a well-defined wall evidenced by radiocontrast; surrounding hypodense area not corresponding to the usual perilesional vasogenic oedema, but defining a well-delimited vascular territory of the right MCA, raising the hypothesis of an ischaemic event; and discrete haemorrhagic cortical densities superimposed to the ischaemic area (figure $1 \mathrm{~A}-\mathrm{C}$ ).

Physical examination was remarkable for the presence of left-sided hemiplegia, a confusional state and low-grade fever. There were no signs of
To cite: Ribeiro $R$, Patrício C, Moura Valejo Coelho $\mathrm{M}$, et al. BMJ Case Rep Published online: [please include Day Month Year] doi:10.1136/bcr-2015212162
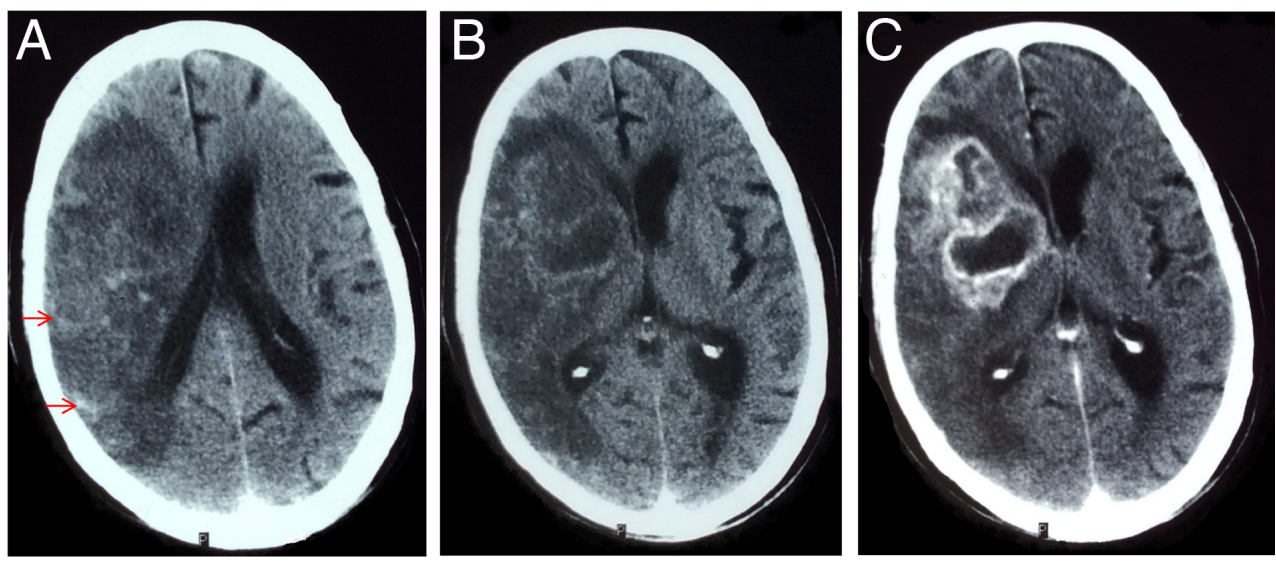

Figure 1 (A) Parenchymal hypodensity of the right medium cerebral artery territory $(A)$ with cortical haemorrhagic densities superimposed in the ischaemic area $(\rightarrow)$. (B) Nucleobasal rounded lesion with mass effect.(C) Multiloculated aspect of the mass with well-defined walls enhanced by radiological contrast. 


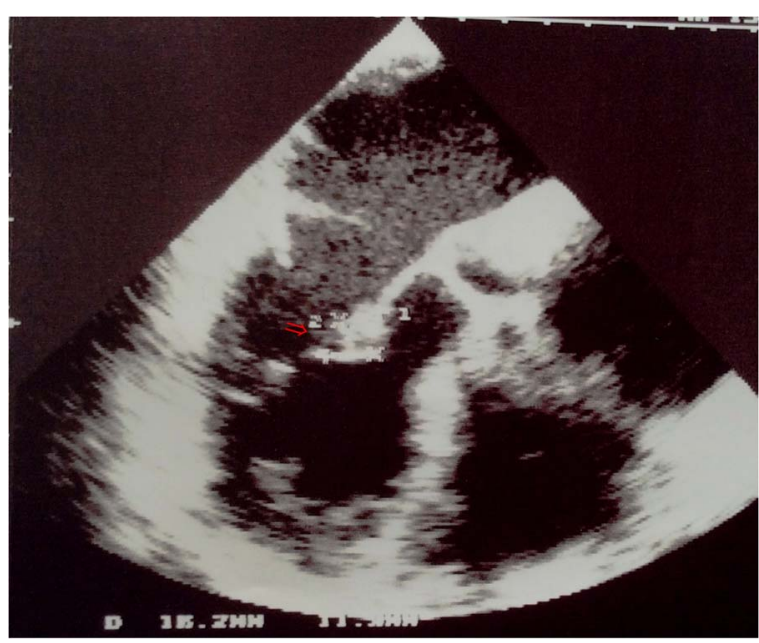

Figure 2 Vegetation of the anterior mitral valve leaflet $(\Rightarrow)$.

sinus ear mastoid or odontogenic infection, meningeal irritation, skin lesions or heart murmurs. No other relevant findings were evident.

\section{INVESTIGATIONS}

Blood analysis revealed: normocytic normochromic anaemia (haemoglobin $11.2 \mathrm{~g} / \mathrm{L}$ ), leucocytosis $16.4 \times 10^{9} / \mathrm{L}$ with $87 \%$ neutrophils, platelets $540 \times 10^{9} / \mathrm{L}, \mathrm{C}$ reactive protein $78 \mathrm{mg} / \mathrm{L}$ (ref: $<5$ ), creatinine $1.0 \mathrm{mg} / \mathrm{dL}$, glycated haemoglobin $5.5 \%$, sodium $135 \mathrm{mEq} / \mathrm{L}$, potassium $3.7 \mathrm{mEq} / \mathrm{L}$, aspartate aminotransferase $37 \mathrm{U} / \mathrm{L}$, alkaline phosphatase $93 \mathrm{U} / \mathrm{L}$, total bilirubin $0.9 \mathrm{mg} / \mathrm{dL}$, lactate dehydrogenase $256 \mathrm{U} / \mathrm{L}$ and serology for HIV negative. ECG showed: sinus rhythm, heart rate $98 \mathrm{bpm}$ and left bundle branch block. A decubitus anteroposterior chest radiograph produced normal findings.

Considering possible haematogenous dissemination, an echocardiography was performed revealing endocarditis of the anterior mitral leaflet (figure 2). Blood cultures (3) and a diagnostic/ therapeutic abscess aspiration were performed, with isolation of E. coli in all the biological samples.

In the face of these results, we decided to ascertain the patient's clinical history by contacting the referring hospital. Seven weeks prior, the patient had been admitted to the emergency department due to acute urinary retention, fever and perineal pain. A bladder indwelling catheter was inserted and urine sample collected for examination and culture; the urinary
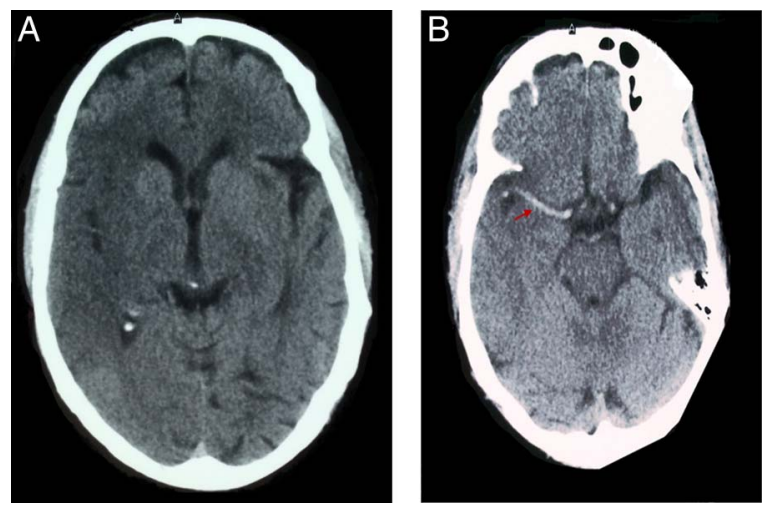

Figure 3 (A) Right medium cerebral artery (MCA) acute ischaemic infarction.(B) Right MCA spontaneous hyperdensity corresponding to an endoluminal thrombus $(\rightarrow)$. sediment showed no signficant signs of infection but still ciprofloxacin was empirically prescribed for a hypothetical prostatitis. Renal ultrasonography at the time excluded urinary tract obstruction. Two weeks later, the patient was admitted with acute left hemiplegia, and the CT scan revealed 'acute ischaemic infarction of the MCA territory with spontaneous hyperdensity of this artery, corresponding to an endoluminal thrombus' (figure 3A, B). E. coli was isolated in the urine sample collected 2 weeks earlier at the emergency department; the antibiogram was identical to the blood and abscess isolates performed at our hospital, including sensitivity to second and third generation cephalosporins.

\section{TREATMENT}

Two CT-guided therapeutic needle aspirations were performed and intravenous antibiotics were empirically started-ceftriax-

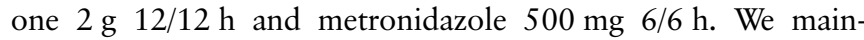
tained these antimicrobials after sensitivity test results were known. Although there was radiological improvement, the patient's neurological deficits and confusional state persisted. Two weeks after starting treatment, he was transferred to the referring hospital to be close to his family.

\section{DISCUSSION}

Brain abscesses can be caused by several different conditions. Only a minority of cases result from direct trauma or neurosurgery. Approximately $40 \%$ of cases result from contiguous spread, including direct extension from infections of the paranasal sinuses, middle ear, mastoid cells and teeth. ${ }^{1}$ Osteomyelitis with subsequent inflammation of the leptomeninges, or septic thrombophlebitis of the intracranial vessels, are the main physiopathological mechanisms. ${ }^{2}$ Around one-third of cases are metastatic, resulting from haematogenous spread in the context of endocarditis or a primary septic focus in the lungs or pleura. Intrapulmonary right-to-left shunting in patients with arteriovenous malformations (Osler-Weber-Rendu syndrome) ${ }^{3}$ and cyanotic congenital heart diseases can also be complicated by cerebral abscess, accounting for a minority of these cases. In about $20-40 \%$, the source cannot be ascertained. ${ }^{4}$

Brain abscesses associated with bacteraemia usually result in multiple abscesses, which are commonly located in the distribution of the MCA. ${ }^{5}$ In our patient, the presence of an abscedated lesion with a surrounding hypodensity corresponding exactly to the MCA territory made us suspect endocarditis as the possible source of septic embolism. Endocarditis is seldom caused by Gram-negative bacteria such as E. coli and Klebsiella pneumoniae, as these microorganisms adhere less readily to heart valves than they do to Gram-positive pathogens. ${ }^{6}$ Also, the most frequent causes of brain abscesses are viridans streptococci and Staphylococcus aureus. ${ }^{7}$ Considering these facts, the microbiological isolation of E. coli in blood as well as in cerebral abscess cultures provided us with a clue about the primary site of infection and a potential undiagnosed underlying condition. When contacting the referring hospital, we obtained some revealing information that enlightened us, as follows:

- A UTI, eventually complicated with prostatitis, had been diagnosed 7 weeks earlier. The urine culture had isolated the same pathogen as had our microbiological examinations: E. coli.

- Bladder catheterisation at that time would have been the initiating event leading to bacteraemia and haematogenous spread of E. coli.

- A septic embolus originating from an endocarditis focus was responsible for the ischaemic MCA stroke 2 weeks later. 
Endocardial seeding of this Gram-negative bacteria may have been facilitated by an eventual age-associated degenerative heart valve disease.

- Secondary cerebral abscedation developed in the ischaemic MCA territory. This corresponds to the lesions detected in the CT scan presented to us 5 weeks later.

\section{Learning points}

- Noticing subtle semiological signs (in this case, a CT scan finding) can modify the global sequence of the clinical reasoning.

- Physicians should correlate facts and search for global explanations in their daily practice. We expect internists to do this proficiently as part of their expertise in the art of approaching the patient.

- When there is no evidence of contiguous/direct spread, one should consider haematogenous dissemination among the possible aetiologies of cerebral abscesses, namely endocarditis.

- The increasing frequency of endocarditis in the elderly runs parallel with the prevalence of degenerative valve disease in this age group. In this context, bacteraemia can easily lead to valvular bacterial seeding.

- Urinary tract infection (UTI) in an elderly man with urinary retention makes it a complicated UTI and not a simple infection. Such infection should be treated aggressively and the end point of treatment should be met by repeating urine culture after the treatment regime is thought to be complete. In elderly male patients, infections such as prostatitis and other complicated UTIs, can result in potentially fatal dissemination. Invasive procedures in this setting are of particular concern.
Successful management of brain abscesses usually requires a combination of antibiotics and some form of surgical drainage for diagnostic as well as therapeutic purposes. ${ }^{8}{ }^{9}$ We performed two CT-guided abscess needle aspirations, and ceftriaxone plus metronidazole was the empirical antibiotic regimen chosen for this patient. Although ceftriaxone would cover the haematogenous-spread of E. coli, we decided to maintain metronidazole, as anaerobes are frequent co-infectious microorganisms in cerebral abscesses.

This case illustrates the importance of collecting data in search for a global explanation, which can reveal less obvious facts from the previous history and modify the future treatment approach.

Competing interests None declared.

Patient consent Not obtained.

Provenance and peer review Not commissioned; externally peer reviewed.

\section{REFERENCES}

1 Yen PT, Chan ST, Huang TS. Brain abscess: with special reference to otolaryngologic sources of infection. Otolaryngol Head Neck Surg 1995;113:15-22.

2 Ropper AH, Brown RH. Adams and Victor's Principles of Neurology. 9th edn. McGraw-Hill, 2009:606-9.

3 Chiu HY, Lin SJ. Red papules on the tongue of a patient with hemiparesis. JAMA 2014;312:741-2.

$4 \mathrm{Ng}$ PY, Seow WT, Ong PL. Brain abscesses: review of 30 cases treated with surgery. Aust N Z J Surg 1995;65:664-6.

5 Bakshi R, Wright PD, Kinkel PR, et al. Cranial magnetic resonance imaging findings in bacterial endocarditis: the neuroimaging spectrum of septic brain embolization demonstrated in twelve patients. J Neuroimaging 1999;9:78-84.

6 Gould K, Ramirez-Ronda CH, Holmes RK, et al. Adherence of bacteria to heart valves in vitro. J Clin Invest 1975;56:1364-70.

7 Brouwer MC, Coutinho JM, van de Beek D. Clinical characteristics and outcome of brain abscesses: systematic review and meta-analysis. Neurology 2014;82:806-13.

8 Arlotti M, Grossi P, Pea F, et al. Consensus document on controversial issues for the treatment of infections of the central nervous system: bacterial brain abscesses. Int J Infect Dis 2010;14(Suppl 4):S79-92.

9 Brouwer MC, Tunkel AR, McKhann GMII, et al. Brain abscess. N Engl J Med 2014:371:447-56.

Copyright 2015 BMJ Publishing Group. All rights reserved. For permission to reuse any of this content visit http://group.bmj.com/group/rights-licensing/permissions.

BMJ Case Report Fellows may re-use this article for personal use and teaching without any further permission.

Become a Fellow of BMJ Case Reports today and you can:

- Submit as many cases as you like

- Enjoy fast sympathetic peer review and rapid publication of accepted articles

- Access all the published articles

- Re-use any of the published material for personal use and teaching without further permission

For information on Institutional Fellowships contact consortiasales@bmjgroup.com

Visit casereports.bmj.com for more articles like this and to become a Fellow 